\title{
Digital Rhetorical Investigation On Filing System And Info Change Of State Professor. Aidan Asgar, Professor. Umar Sayed
}

\author{
Computer Engineering Department International Islamic University Malaysia
}

\begin{abstract}
Digital rhetoricals is that the identification, extraction, analysis and documentation of digital proof from storage media. it's comparatively new technology that is more and more turning into vital because the criminals sharply expand the utilization of technology. Digital data is fragile and it are often simply changed or destroyed like filing system and info change of state. within the course of the investigation, the investigator ought to assure that digital evidences aren't changed unauthorized and demonstrate submission within the court of law. Our paper explains rhetorical investigation procedures employing a WinHex tool[10]. Main focus of our paper is digital rhetorical investigation of various locations of windows filing system and oracle info area unit explained. proof assortment from hidden locations of windows filing system and oracle $10 \mathrm{~g}$ info can facilitate the investigators in trusting and thorough investigation.
\end{abstract}

Keywords -law-breaking, Digital forensic, filing system rhetorical, info Investigation.

\section{Introduction}

Computers area unit integral a part of our life. a big proportion of today's transactions and processes turn up mistreatment laptop and web. folks have pronto adopted this technology and have innocently trusty it whereas playing several tasks, with cognitive content concerning the restrictions and threats to their securities. With this advance in technology, associate equally advanced style of crimes has emerged. differing types of cyber attacks from numerous sources could adversely have an effect on computers, software, a network, associate agency's operations, associate business, or the net itself. therefore the corporations and product aim to require help of legal and laptop Forensics. Digital forensics is that the science of distinctive, extracting, analyzing and presenting the digital proof that has been hold on within the digital devices. numerous digital tools and techniques area unit getting used to realize this. rhetorical tools and techniques area unit integral a part of criminal investigations wont to work suspect systems, gathering and conserving proof, reconstructing events, and assessing the present state of an 


\section{THE AMERICAN JOURNAL OF}

ENGINEERING AND TECHNOLOGY

incident. This paper organized into 2 sections initial section explains however a criminal offense is work. Second section explains for collection proof from a filing system and info. Digital rhetorical Science covers Storage Media forensics, Network forensics, Firewall forensics, Device forensics, info forensics, Mobile device forensics, software package forensics, live systems forensics etc. during this paper we have a tendency to make a case for Storage media Forensics and network rhetorical.

\section{Cyber - Crime Investigation}

A. Investigation methodes the whole investigation process are often divided into four phases.

1. Identification: during this section it collects the data of the compromised system. System Configuration, software package loaded, User profiles etc

2. assortment Phase; Collects the proof from the compromised system. proof is most ordinarily found in files and Databases that area unit hold on on onerous drives and storage devices and media. If file deleted, convalescent knowledge from the deleted files and additionally collects proof file deleted files.

3. Analysis phase: Analyse the collection data/files and searching for the particular proof.

4. Report phase: The audience are going to be ready to perceive the proof knowledge that has been nonheritable from the proof assortment and analysis phases.

Storage media Forensics. A. Basic Steps in storage media investigation.

1. Replication of rhetorical image: - Nonintrusive acquisition of a replicated image of information extracted from the questioned device. 


\section{THE AMERICAN JOURNAL OF}

ENGINEERING AND TECHNOLOGY

2. For integrity perform Hash worth calculation.

3. Conducting a file-fragment recovery procedure to recover files and folders to a replacement location.

4. Examine all files particularly deleted files

5. Reviewing typical evidentiary objects such as:

a. Analyse free areas, slack areas and dangerous sectors

b. Application software package file.

c. camera, printer and auxiliary devices.

d. E-mails, Games \& Graphics pictures

e. web chat logs \& Network activity logs

f. Recycle folders

g. System and file date / time objects

h. User-created directories, folders, and files

i. Latent knowledge extraction from page, temp, and written account area.

6. Copy the content of the evidentiary object into text files.

7. looking for key-term strings.

8. Reviewing file notations.

\section{Hidden knowledge analysis in storage media}

Suspects will hide their sensitive knowledge in numerous areas of the filing system like Volume slack; file slack, dangerous clusters, deleted file areas. [16] 


\section{THE AMERICAN JOURNAL OF}

ENGINEERING AND TECHNOLOGY

Hard disk: the upkeep track / Protected space on ATA disks area unit wont to hide data. The proof assortment tools will copy the higher than contents.

File System Tables: A file allocation table in FAT and main file Table in NTFS area unit wont to keep track of files. These entries area unit manipulated to cover important and sensitive data.

\section{File Deletion}

once a file is deleted, the record of the file is off from the table, thereby creating it seem that it doesn't exist any longer. The clusters employed by the deleted file area unit marked as being free and may currently be wont to store different knowledge. However, though the record is gone, the info should reside within the clusters of the magnetic disk. That knowledge we are able to recover by calculate beginning and finish of the get in Hex format and duplicate it into a computer file and save with corresponding extension.

\section{info Forensics}

Database Forensics could be a branch of digital rhetorical science regarding the rhetorical study of databases and their connected data. A rhetorical examination of a info could relate to the timestamps that apply to the update time of a row in an exceedingly relative table being inspected and tested for validity so as to verify the actions of a info user. instead, a rhetorical examination could specialise in distinctive transactions inside a info system or application that indicate proof of wrong doing, like fraud.

\section{Conclusion}

There area unit many ways of securing the info and filing system. The attackers have the ways to violate the safety. Then comes the role of rhetorical analyst United Nations agency ought to have a radical data of the fundamentals of a info and filing system and additionally the data concerning constant on that he's getting to perform the analysis. The rhetorical analyst ought to even be ready to suppose from the attacker's purpose of read. supported completely different cases, the digital evidences are often collected from the desired locations. If the intensions of the wrongdoer area unit noted distinctive the attacked location could also be easier. Thinking from the attacker's purpose of read this paper offers a contribution towards the identification of the 
overall locations in an exceedingly info and therefore the filing system for collection the digital evidences

\section{References}

1. K. Hellany \& M. Nagrial. Network Security Approach for Digital Forensics Analysis 2005 IEEE.

2. K. Brannon, and Thomas Song laptop rhetoricals: Digital Forensic Analysis Methodology. laptop Forensics Journal January 2004.

3. Cheong Kaiwee. Analysis of Hidden knowledge in NTFS filing system. Whitepaper.

4. Mamoun, Alazab, Sitalakshmi Venktraman, Paul Watters. Effective Digital rhetorical Analysis of the NTFS Disk Image.

5. R. Leschke. Cyber Dumpster-Diving: \$Recycle.Bin Forensics for Windows seven and Windows visual image.

6. Keith J. Jones rhetorical Analysis of Microsoft Windows Recycle Bin Records.

7. office Qinquan, Wu shunxiang. analysis of Recycle Bin rhetorical Analysis Platform supported XML Techniques. 